\title{
Förderung von unterrichtsbegleitendem Diagnostizieren von Schülervorstellungen durch Video- und Textvignetten
}

\author{
Tobias Hoppe (D) Alexander Renkl • Werner Rieß
}

Online publiziert: 3. Juni 2020

(C) Der/die Autor(en) 2020

Zusammenfassung Fachlich wenig anschlussfähige Schülervorstellungen können den Aufbau fachlich gültiger ökologischer Konzepte im Biologieunterricht beeinträchtigen, wenn Lehrkräfte diese im Unterrichtsverlauf nicht diagnostizieren und angemessen darauf reagieren. Unterrichtsbegleitende Diagnosen von Äußerungen der Schülerinnen und Schüler müssen häufig spontan (,on-the-fly“) erfolgen. Es wäre wünschenswert, bereits angehende Lehrkräfte auf diese herausfordernde Aufgabe vorbereiten zu können. In einem experimentellen Prä-Posttest-Design $(N=36)$ wurden in dieser Studie Einflüsse auf den Erwerb von Fertigkeiten zur Diagnose von Schülervorstellungen untersucht. Hierfür wurden verschiedene Merkmale fallbasierter Lernsettings systematisch variiert (Video vs. Text/unterschiedliche Arten von Schülervorstellungen). Ein Training an Fallmedien erwies sich für bestimmte Diagnoseinhalte als effektiv im Vergleich zu einer Vermittlung diagnostischen Wissens ohne fallbasiertes Training. Unerheblich schien zu sein, ob als Fallmedium Video- oder Textvignetten verwendet wurden. Darüber hinaus zeigte sich, dass die Fertigkeiten zur Diagnose im Wesentlichen inhaltsspezifisch erworben werden. Die Ergebnisse werden hinsichtlich der Entwicklung von Fördermaßnahmen in der Lehrerbildung diskutiert.

Schlüsselwörter Diagnostische Fertigkeiten · Schülervorstellungen ·

Videovignetten · Textvignetten · „on-the-fly“-Diagnostik

T. Hoppe $(\bowtie) \cdot$ W. Rieß

Institut für Biologie und ihre Didaktik, Pädagogische Hochschule Freiburg,

Kunzenweg 21, 79117 Freiburg, Deutschland

E-Mail: tobias.hoppe@ph-freiburg.de

A. Renkl

Institut für Psychologie, Albert-Ludwigs-Universität Freiburg, Engelbergerstr. 41, 79085 Freiburg,

Deutschland 


\title{
Fostering on-the-fly judgements of students' conceptions using video and text vignettes
}

\begin{abstract}
Students' intuitive conceptions can inhibit the acquisition of scientifically correct ecological concepts in biology lessons if teachers do not diagnose these conceptions and address these adaptively. During instruction, teachers often have to judge students' expressions spontaneously ("on-the-fly"). Future teachers should be prepared for this challenging task. In this study, influences on the acquisition of diagnostic skills have been examined within an experimental pretest-posttest design $(N=36)$. For this purpose, various characteristics of case-based learning environments have been systematically varied (video vs. text/different kinds of students' conceptions). Trainings of diagnostic skills using case media appeared to be effective as compared to the presentation of diagnostic knowledge without a training opportunity. Using video or text as case media had not made a significant difference. Furthermore, the acquisition of diagnostic skills in this particular area seemed to be content-specific. Our findings are discussed with regard to developing instructional support procedures in teacher education.
\end{abstract}

Keywords Diagnostic skills · Students' intuitive conceptions · Video vignettes · Text vignettes · "on-the-fly" assessment

\section{Einleitung}

Die Aneignung fachlicher Konzepte kann durch schon vorhandene Vorstellungen der Schülerinnen und Schüler zu den Unterrichtsgegenständen erschwert werden, wenn es Lehrkräften nicht gelingt diese aufzugreifen (Schrenk et al. 2019). Das Aufgreifen kann auf der einen Seite planvoll erfolgen, in dem in der Literatur dokumentierte Vorstellungen bereits bei der Unterrichtsplanung bedacht und/oder eine Diagnostik vorbereitet wird (z.B. Kattmann 2015). Auf der anderen Seite müssen Lehrkräfte aber auch im Unterrichtsverlauf geäußerte Schülervorstellungen wahrnehmen und interpretieren können, um angemessen darauf zu reagieren (Shavelson et al. 2008). In dieser Studie geht es um letztere Diagnosen, welche spontan - also ,on-the-fly“ - erfolgen. Zusätzlich zur allgemeinen Komplexität des Unterrichtsgeschehens stellt dabei die Flüchtigkeit der Situation eine besondere Herausforderung dar (Knievel et al. 2015).

Will man Fertigkeiten zu ,on-the-fly“-Diagnosen bereits in der ersten Phase der Lehramtsausbildung fördern, stellt sich die Frage, in welcher Weise dies effektiv geschehen kann. Der Einsatz von Fallmedien hat in der Lehrerbildung bereits eine längere Tradition (Gaudin und Chaliès 2015). Vorliegende Studien zur Wirksamkeit der Fallarbeit beziehen sich in den meisten Fällen auf das Lernen mit Unterrichtsvideos (Syring et al. 2015). Dabei bleibt offen, inwieweit der Einsatz von Videos einen Mehrwert im Vergleich zu anderen Vermittlungsmöglichkeiten hat (Sunder et al. 2016). Insbesondere mit Bezug zu fachdidaktischen Aspekten von Unterricht finden sich kaum Studien, die z. B. systematisch den Einsatz unterschiedlicher Fallmedien als Förderinstrumente vergleichen (vgl. Chernikova et al. 2019). 
Für eine Förderung von Fertigkeiten zur Diagnose von Schülervorstellungen ist von Interesse, welchen Einfluss der Diagnosegegenstand (unterschiedliche Schülervorstellungen) und das Fallmedium auf die Diagnosefertigkeiten und deren Erwerb haben. In der vorliegenden Studie wurde im Rahmen einer Intervention zur Förderung von Diagnosefertigkeiten variiert, welche Fallmedien eingesetzt wurden (Video bzw. Text) und welche biologischen Inhaltsbereiche die Schülervorstellungen betreffen. Einer Kontrollgruppe wurde Wissen über die Schülervorstellungen ohne ein Training an Fallmedien vermittelt. Das DiaCoM-Rahmenmodell von Loibl et al. (2020) bietet die Möglichkeit, die beim Diagnostizieren zugrundeliegenden kognitiven Mechanismen als Informationsverarbeitungsprozesse zu deuten.

\section{Schülervorstellungen und deren unterrichtsbegleitende Diagnose}

\subsection{Die Bedeutung von Schülervorstellungen für das Lernen}

Schülervorstellungen sind eine wichtige Lernausgangslage (Schrenk et al. 2019) und sind damit im naturwissenschaftlichen Unterricht ein bedeutsamer Diagnosegegenstand (Rath 2017). Mit Schülervorstellungen sind die bereits vorhandenen Vorstellungen von Lernenden zu naturwissenschaftlichen Phänomenen, Begriffen und Prinzipien gemeint (Gebhard 2013; Häußler 1998). Den fachlich gültigen Vorstellungen entsprechen Schülervorstellungen häufig nicht oder nur teilweise (Kattmann 2015; Schecker und Duit 2018).

Für den Themenbereich der Stoffkreisläufe konnte beispielsweise übereinstimmend und über unterschiedliche Altersgruppen hinweg nachgewiesen werden, dass von vielen Lernenden in erster Linie abiotische Faktoren (z. B. Wind, Wärme, Frost) als Ursache für Zersetzungsprozesse angesehen werden, während die ursächliche Bedeutung von Mikroorganismen nicht als Erklärung genannt wird (Baisch 2009; Leach et al. 2008). Solche Vorstellungen entstehen meist aufgrund von Alltagserfahrungen der Lernenden (Hammann und Asshoff 2015; Häußler 1998) oder aufgrund von vorangegangenem Unterricht (Schrenk et al. 2019).

Für die Planung und Durchführung naturwissenschaftlichen Unterrichts sind Schülervorstellungen deswegen so bedeutsam, weil sie das Lernen entscheidend beeinflussen: Die Konstruktion neuen Wissens erfolgt auf Grundlage vorhandener Vorstellungen (Riemeier 2007). Gleichzeitig haben sich Schülervorstellungen als änderungsresistent erwiesen (Duit et al. 2008) bzw. sie verändern sich oft nicht so, wie es Lehrkräfte beabsichtigen (Zabel und Gropengießer 2011). In den Naturwissenschaftsdidaktiken besteht ein breiter Konsens darüber, dass Schülervorstellungen zum Ausgangspunkt des Unterrichts gemacht werden sollen (z. B. Kattmann 2017; Schrenk et al. 2019) - insbesondere bei Stoffgebieten, in denen es viele wissenschaftlich nicht haltbare Schülervorstellungen gibt (Schecker und Duit 2018). Um mit im Unterricht aktuell auftretenden Schülervorstellungen umzugehen, müssen Lehrkräfte diese spontan wahrnehmen und interpretieren können (Shavelson et al. 2008). 


\subsection{Unterrichtsbegleitende Diagnosen von Schülervorstellungen als Ziel pädagogische Diagnostik}

Mit dem Ziel einer adaptiven Unterrichtsgestaltung werden Schülervorstellungen zu einem zentralen Gegenstand unterrichtsbegleitender pädagogischer Diagnostik (Beck et al. 2008; Klieme und Warwas 2011; Ruiz-Primo und Furtak 2006). Pädagogische Diagnostik kann u.a. als Prozess des Einschätzens lernrelevanter Schülermerkmale verstanden werden (Herppich et al. 2017a). Gelegenheiten zur Einschätzung von Schülervorstellungen bieten insbesondere verbale Äußerungen der Schülerinnen und Schüler (Rath 2017), beispielsweise wenn Lehrpersonen bei Gruppenarbeiten Erklärungen der Lernenden mithören (Shavelson et al. 2008) oder wenn Lernende zum Unterrichtsgespräch beitragen (Ruiz-Primo und Furtak 2007).

Unterrichtsbegleitende Diagnosen können auch geplant verlaufen, z.B. wenn Lehrpersonen sich vorab eine lernzielorientierte Frage überlegen und diese in der Interaktion mit den Schülerinnen und Schülern stellen (Glogger-Frey und Herppich 2017). Neben dieser geplanten Diagnostik, sollten Lehrpersonen Schülervorstellungen auch diagnostizieren können, wenn sie die Schüleräußerungen nicht bewusst hervorgerufen haben. Wenn Prozesse des Diagnostizierens von Schülervorstellungen spontan und unmittelbar erfolgen, werden diese Einschätzungen als „on-thefly“-Diagnosen bezeichnet (Glogger-Frey und Herppich 2017; Klieme und Warwas 2011; Shavelson et al. 2008). „On-the-fly“-Diagnosen sind für Lehrkräfte nur eingeschränkt planbar. Zudem stellt die Flüchtigkeit der diagnostischen Situation eine besondere Herausforderung für Lehrkräfte dar (Knievel et al. 2015).

Dass unterrichtsbegleitende Diagnosen von Schülervorstellungen in Verbindung mit Adaptionen des Unterrichts das Lernen der Schülerinnen und Schüler förderlich beeinflussen können, ist theoretisch sehr plausibel (Rath 2017). In einigen Studien konnte für den naturwissenschaftlichen Unterricht auch empirisch gezeigt werden, dass sich entsprechende Unterrichtspraktiken, d. h. ein unterrichtsbegleitendes Diagnostizieren von individuellen Lernständen und entsprechende Unterrichtsadaptionen, positiv auf den Lernzuwachs der Schülerinnen und Schüler auswirken (Decristan et al. 2015; Furtak et al. 2016a; Ruiz-Primo und Furtak 2007). Angesichts der Herausforderung, die solche Unterrichtspraktiken mit sich bringen, stellt sich die Frage, wie diagnostische Fertigkeiten bereits bei angehenden Lehrkräften gefördert werden können (Dannemann et al. 2018; Ruiz-Primo 2011).

\subsection{Konzeptualisierung von kognitiven Fertigkeiten zur unterrichtsbegleitenden Diagnose von Schülervorstellungen}

Für adaptives Unterrichtshandeln ist die Güte des diagnostischen Urteils über den aktuellen Lernstand der Schülerinnen und Schüler maßgebend (Brühwiler 2017). Ausgehend von den diagnostischen Urteilen können Lehrkräfte für pädagogische Handlungen Konsequenzen ableiten; z. B. Feedback an die Lernenden (z. B. von Aufschnaiter et al. 2018; Furtak et al. 2016a) oder auch weitere diagnostische Aktivitäten durchführen; z. B. Rückfragen an die Lernenden stellen, wenn eine Lehrkraft das eigene Verständnis über die Schülervorstellungen erweitern möchte (z. B. Heitzmann et al. 2019; Herppich et al. 2017b; Van de Pol et al. 2014). Insofern stellt 
der Erwerb von Fertigkeiten zur Urteilsbildung ein spezifisches, aber wesentliches Element bei der Förderung diagnostischer Kompetenzen von Lehrkräften dar.

Das unterrichtsbegleitende, spontane Diagnostizieren von Schülervorstellungen in Schüleräußerungen kann verstanden werden als ein andauerndes Filtern von Informationen, um daraus diagnostische Urteile in Form von Hypothesen zu generieren (Heitzmann et al. 2019). Die kognitiven Mechanismen, welche der Verarbeitung jener Informationen unterliegen, können als Informationsverarbeitungsprozesse der Wahrnehmung und Interpretation konzeptualisiert werden (Blömeke et al. 2015; Loibl et al. 2020). Wahrnehmung bedeutet hier, dass im Fluss der Aussagen im Sinne eines thematisch selektiven Erkennens (Blomberg et al. 2011) diejenigen Aussagen identifiziert werden, deren Interpretation für ein Verständnis der zugrundeliegenden Schülervorstellungen wichtig sein könnte. Interpretation meint in diesem Kontext, inwiefern wahrgenommene Schüleräußerungen hinsichtlich eines Verständnisses der dahinterliegenden Schülervorstellungen gedeutet werden.

In Anlehnung an Blömeke et al. (2015), können die kognitiven Prozesse des Wahrnehmens und Interpretierens als situationsspezifische, kognitive Fertigkeiten betrachtet werden. Diese vermitteln zwischen kognitiven Dispositionen (z. B. Fachwissen und fachdidaktisches Wissen) bzw. affektiv-motivationalen Dispositionen (z.B. Interesse, Einstellungen) und der Performanz der Lehrkräfte. Eine Förderung der situationsspezifischen, kognitiven Diagnosefertigkeiten des Wahrnehmens und Interpretierens bezieht sich entsprechend auf einen spezifischen Anteil eines umfassenderen Konstrukts diagnostischer Kompetenz.

\subsection{Kriterien für die Beurteilung des Niveaus kognitiver Fertigkeiten zur unterrichtsbegleitenden Diagnose von Schülervorstellungen}

Das Fertigkeitsniveau bei der Urteilsbildung ergibt sich aus dem Zusammenspiel der beiden oben beschriebenen Teilfertigkeiten des Wahrnehmens und Interpretierens (Sherin et al. 2011) und zeigt sich in Verhaltensaspekten der diagnostizierenden Lehrkräfte; z.B. Verbalisierung des diagnostischen Urteils, Rückmeldung an die Lernenden (Loibl et al. 2020). Ein hohes Fertigkeitsniveau zeichnet sich einerseits dadurch aus, dass relevante Schüleräußerungen als bedeutsam für eine Einschätzung der Lernenden wahrgenommen werden. Andererseits ist die Angemessenheit der Interpretation ausschlaggebend. Wenn die Ausprägungen der hier beschriebenen Diagnosefertigkeiten empirisch untersucht und beschrieben werden, dann handelt es sich um eine Rekonstruktion der kognitiven Prozesse. Von den vorliegenden Daten wird auf die vermuteten Informationsverarbeitungsprozesse des Wahrnehmens und Interpretierens geschlossen (Furtak et al. 2016b). Wenn Daten z. B. in Form verbalisierter Antworten vorliegen, beziehen sich die Rückschlüsse zudem eher auf das Produkt einzelner Prozesse (z. B. Interpretieren) als auf die Prozesse selbst (Loibl et al. 2020).

Für die Beurteilung der Interpretationsleistung lassen sich sowohl inhaltliche als auch formale Kriterien theoretisch begründen. Aus fachdidaktischer Perspektive ist es unzureichend, wenn fachlich nicht gültige Aussagen der Lernenden lediglich als abweichend von einer wissenschaftlich korrekten Darstellung gekennzeichnet werden. Um Schülerinnen und Schülern aussichtsreiche Lernwege zu ermöglichen, gilt 
es vielmehr, aus den Schüleräußerungen die dahinterliegenden spezifischen Vorstellungen zu erschließen und die Denkweisen zu verstehen (Furtak et al. 2016a; Gropengießer und Marohn 2018; Schrenk et al. 2019). Im Rahmen der fachdidaktischen Vorstellungsforschung wurden Schülervorstellungen zu vielen biologischen Themen empirisch erhoben und hinsichtlich ihrer gedanklichen Konstruktion beschrieben (z. B. Kattmann 2015).

Nach Helmke (2017) liegt formal ein charakteristisches Merkmal des Diagnostizierens darin, anhand vorgegebener Kategorien, Begriffe oder Konzepte zu urteilen (vgl. dazu auch Heitzmann et al. 2019). Prinzipiell können Kategorien bei der Diagnose von Schülervorstellungen sowohl induktiv, also von den Schüleräußerungen her, als auch deduktiv, d.h. von Forschungsergebnissen ausgehend gebildet werden (z.B. Hammann und Jördens 2014). Da Lernende oft ähnliche Vorstellungen zu bestimmten Themen haben, ist es beim Diagnostizieren von Schülervorstellungen für die Lehrkräfte tatsächlich in vielen Fällen möglich, auf der Grundlage von Forschungsergebnissen gezielte Hypothesen zu den Vorstellungen der Lernenden zu bilden (Taber 2017). Auf diese Weise dürfte eine zielgerichtete und zuverlässige Deutung der Vorstellungen der eigenen Schülerinnen und Schüler eher gelingen (Gropengießer 1996; Kattmann 2015). Vor dem Hintergrund, dass insbesondere informelle Diagnoseleistungen als fehleranfällig gelten (Schrader 2013), erscheint eine hypothesengeleitete Interpretation anhand bekannter Schülervorstellungen insbesondere für angehende Lehrkräfte bzw. Lehrkräfte mit wenig Erfahrung als vorteilhaft. Darüber hinaus ermöglicht eine Zuordnung von Schüleräußerungen zu bekannten Schülervorstellungen, sich bei pädagogischen Entscheidungen an entsprechenden Vorschlägen für spezifische Instruktionsstrategien zu orientieren (z.B. Kattmann 2015). Zusammenfassend zeigt sich ausgehend von den hier aufgeführten theoretischen Überlegungen eine gute Interpretationsleistung bei der Bildung diagnostischer Urteile darin, dass ausgehend von einer Analyse der Denkweise der Lernenden die (vermutete) Vorstellung möglichst präzise kategorisiert und benannt werden kann (vgl. Grossman et al. 2009).

\subsection{Wie können Fertigkeiten zur Diagnose von Schülervorstellungen gefördert werden?}

\subsubsection{Welche Wirkungen hat der Einsatz unterschiedlicher Fallmedien auf den Erwerb von Diagnosefertigkeiten?}

Beim Diagnostizieren fällt es Studierenden und Berufseinsteiger/-innen oft schwer, ihr Wissen in komplexen Unterrichtssituationen auf konkrete Fälle anzuwenden (Heitzmann et al. 2019). Um bei Lehramtsstudierenden kognitive Fertigkeiten in Bezug auf das Diagnostizieren von Schülervorstellungen in flüchtigen Unterrichtssituationen zu fördern, sollte eine Lernbedingung gewählt werden, die sich der unterrichtlichen Situation in wichtigen Facetten annähert und gleichzeitig nicht überfordert (Grossman et al. 2009, 2018). Das Lernen an Fällen ermöglicht es, Anforderungen des beruflichen Alltags zu veranschaulichen und Fertigkeiten zur Beurteilung und Reflexion von Praxissituationen zu fördern (Krammer 2014). Fallbasiertes Lernen 
wird in der Lehrerbildung vor allem anhand von Video- und Textfällen realisiert (Schneider et al. 2016).

Die Analyse von Videofällen hat sich in der Lehrerbildung grundlegend bewährt (Gaudin und Chaliès 2015). Videos bilden viele Facetten der unterrichtlichen Situation ab (z. B. Flüchtigkeit der Situation, Mimik und Gestik der Lernenden und Lehrkräfte). Aufgrund dieser Nähe zur komplexen unterrichtlichen Realität gelten Videofälle als besonders authentisch. Allerdings sind sie damit eine komplexe Vorlage und stellen hohe Anforderungen an die Informationsverarbeitungskapazitäten von angehenden Lehrkräften dar (Syring et al. 2015). Die relative Flüchtigkeit der Information in Videos im Vergleich zu Textfällen kann einen Einfluss auf die Prozesse der Informationsverarbeitung haben (Leahy und Sweller 2011; Loibl et al. 2020). Textfälle (z.B. Transkripte) ermöglichen insofern eine Komplexitätsentlastung (Reh und Schelle 2010). Sie gelten allerdings als weniger authentisch, da sie weniger Facetten der unterrichtlichen Realität abbilden (Schutz et al. 2018).

Hinsichtlich der Möglichkeiten und Einschränkungen des Einsatzes beider Fallmedien stellt sich die Frage, inwieweit sich der Einsatz von Text- oder Videofällen als vorteilhaft bei der Förderung kognitiver Fertigkeiten in Bezug auf die Diagnose von Schülervorstellungen erweist. Einige Studien weisen darauf hin, dass Text- und Videofälle ein ähnliches Potential bei der Förderung bestimmter Lehrerkompetenzen haben (z.B. Kramer et al. 2017; Schneider et al. 2016; zur Wahrnehmung und Interpretation in Bezug auf Classroom Management), wobei bei Schneider et al. die Textgruppe für den Teilaspekt der Qualität der Interpretation eine bessere Leistung zeigte als die Videogruppe. Die Autoren führen dies auf die höhere kognitive Belastung durch die Unterrichtsvideos zurück (Schneider et al. 2016).

Studien, bei denen verschiedene Fallmedien (z. B. Video- und Textvignetten) als Förderinstrumente in der Lehrerbildung verglichen wurden, beziehen sich bislang weitgehend auf die Förderung professioneller Unterrichtswahrnehmung im Bereich der Klassenführung (z.B. Kramer et al. 2017; Schneider et al. 2016; Syring et al. 2015). Ob sich eine Förderung mithilfe von Text- oder Videofällen effektiver auf den Erwerb von gegenstandsspezifischen Diagnosefertigkeiten (hier: Diagnostizieren von Schülervorstellungen) auswirkt, bleibt weitgehend offen.

\subsubsection{Welche Bedeutung hat der Diagnosegegenstand für den Erwerb von Diagnosefertigkeiten?}

Es wird angenommen, dass die Diagnosegüte in Abhängigkeit der einzuschätzenden Merkmale variiert (Schrader und Praetorius 2018; Spinath 2005). Insofern sind mit dem Ziel der Entwicklung spezifischer Fördermaßnahmen auch die Effekte auf den Erwerb von Diagnosefertigkeiten, welche von unterschiedlichen Eigenschaften des Diagnosegegenstands möglicherweise ausgehen, zu berücksichtigen.

Bei Schülervorstellungen als Diagnosegegenstand liefern verbale Äußerungen der Lernenden wesentliche Indizien (Schecker und Duit 2018). In den Äußerungen zum Vorschein kommende Schülervorstellungen unterscheiden sich u. a. hinsichtlich des Diagnoseinhalts, z. B. themenübergreifende Phänomene (z. B. Anthropomorphismen) oder spezielle Themen wie bestimmte Bereiche in der Ökologie (Stoffkreisläufe, Ökosysteme, Auswirkungen des Klimawandels etc.) oder Humanbiologie (Ham- 
mann und Asshoff 2015). Darüber hinaus unterscheiden sich Schülervorstellungen auch hinsichtlich der Komplexität ihrer gedanklichen Konstruktion (Gropengießer 2006).

Die Relevanz bestimmter Wissensbestände auf Seiten der Lehrkräfte (u. a. Fachwissen und fachdidaktisches Wissen) für den Erwerb von Diagnosefertigkeiten ist unbestritten (z. B. Baumert und Kunter 2006). Rath (2017) konnte für die Diagnose physikalischer Schülervorstellungen neben der Bedeutung von Fachwissen die Relevanz spezifischen fachdidaktischen Wissens (hier: Wissen über spezifische Schülervorstellungen) nachweisen. Aufgrund der Vielzahl zu diagnostizierender Schülervorstellungen stellt sich die Frage, inwiefern diagnostische Fertigkeiten inhaltsbezogen, d. h. bezogen auf inhaltlich spezifische Vorstellungen, erworben werden oder diese Fertigkeiten dann auch in anderen Inhaltsbereichen ein verbessertes diagnostisches Urteilen ermöglichen.

\subsection{Ziele der Studie und Forschungsfragen}

Bisher gibt es wenig empirische Evidenz darüber, wie der Erwerb gegenstandsspezifischer diagnostischer Fertigkeiten bezogen auf das unterrichtsbegleitende Diagnostizieren von Schülervorstellungen gefördert werden kann. Eine Förderung von Diagnosefertigkeiten mithilfe von Fallmedien bietet die Möglichkeit einer komplexitätsreduzierten Annäherung an die unterrichtliche Situation. Es ist zu klären, inwiefern der Einsatz von Fallmedien den Erwerb von Diagnosefertigkeiten begünstigt und ob sich ein bestimmtes Fallmedium (hier: Video oder Text) effektiver auf den Erwerb von Diagnosefertigkeiten auswirkt. Theoretisch ist es plausibel, dass sich auch unterschiedliche Eigenschaften des Diagnosegegenstands (hier: unterschiedliche Inhaltsbereiche der Schülervorstellungen) auf den Erwerb von Diagnosefertigkeiten auswirken. Aufgrund der Vielzahl möglicher Schülervorstellungen ist es von großem praktischen Interesse, inwiefern Diagnosefertigkeiten inhaltsbezogen oder über unterschiedliche Inhaltsbereiche hinweg erworben werden.

Ziel der Studie war es, zur Klärung dieser Forschungslücken bezüglich des Erwerbs von Diagnosefertigkeiten durch eine systematische Variation von Fördermedien und Diagnosegegenständen beizutragen. Dabei standen folgende drei Forschungsfragen im Fokus:

Forschungsfrage 1 Führt ein Training an Fallmedien in Kombination mit der Vermittlung spezifischen fachdidaktischen Wissens zu einer Verbesserung diagnostischer Fertigkeiten im Vergleich zu einer Kontrollgruppe, in der nur spezifisches fachdidaktisches Wissen vermittelt wird?

Forschungsfrage 2 Lassen sich diagnostische Fertigkeiten mit Video- oder Textvignetten wirksamer fördern?

Forschungsfrage 3 Werden diagnostische Fertigkeiten mithilfe von vignettenbezogenen Fördermaßnahmen inhaltsspezifisch erworben oder erfolgt auch eine Sensibilisierung für einen anderen Inhaltsbereich? 
Tab. 1 Experimentalgruppen und Kontrollgruppe $(N=36)$

\begin{tabular}{llll}
\hline Gruppe (UV) & $n$ & Fallmedium & Schülervorstellungen \\
\hline Video_BP & 8 & Video & BP \\
Text_BP & 7 & Text & BP \\
Video_SZ & 8 & Video & SZ \\
Text_SZ & 6 & Text & SZ \\
Kontrollgruppe & 7 & - & BP und SZ \\
\hline
\end{tabular}

\section{Methode}

\subsection{Stichprobe und Design}

An der Untersuchung nahmen insgesamt $N=36$ Lehramtsstudierende der Biologie (23 weiblich) teil. Die Studie fand im April 2018 im Rahmen eines Kompaktseminars statt. Das Alter der Studierenden lag zwischen 20 und 34 Jahren $(M=23,37$ Jahre; $S D=3,55)$. In der Stichprobe waren die Studiengänge des Lehramts für die Primarund Sekundarstufe I vertreten (7 Primarstufe). Die Studierenden befanden sich zum Zeitpunkt der Studie durchschnittlich im vierten Fachsemester $(M=4,4 ; S D=1,45)$. Alle Studierenden haben zum Zeitpunkt der Intervention ein fachwissenschaftliches Grundlagenstudium im Fach Biologie durchlaufen. Die Benotung der entsprechenden Modulklausur lag im Durchschnitt bei $M=2,8(S D=0,66)$. Schülervorstellungen zur Ökologie sind zuvor nicht Gegenstand im Studium gewesen. Alle Studierenden hatten bereits Unterrichtserfahrung in Form der Teilnahme an einem Orientierungspraktikum (OSP) gesammelt.

Die Studie folgte einem experimentellen Prä-Posttest-Design. Die Lehramtsstudierenden wurden randomisiert den Experimentalbedingungen zugewiesen (s. Tab. 1). In den Experimentalbedingungen wurden für die Intervention die Fallmedien (Video vs. Text) und die Eigenschaften der Diagnosegegenstände (Schülervorstellungen aus zwei Inhaltsbereichen innerhalb eines übergeordneten biologischen Themas) systematisch variiert. Alle verwendeten Schülervorstellungen bezogen sich auf ökologische Basiskonzepte aus dem übergeordneten Themenbereich der Stoffkreisläufe. Die Schülervorstellungen betrafen entweder die Bedeutung von Pflanzen in Ökosystemen (BP) oder die Stoffzersetzung (SZ). Der Kontrollgruppe wurden fachdidaktische Inhalte zu denselben Schülervorstellungen vermittelt, sie nahm aber nicht an einer Intervention mithilfe von Fallmedien teil.

\subsection{Materialien zur Förderung diagnostischer Fertigkeiten}

Um die Diagnosefertigkeiten der Lehramtsstudierenden zu trainieren, wurden für die Intervention zu jedem Inhaltsbereich (BP und SZ) drei Videofälle in Orientierung an Manuals zur Produktion geskripteter Videovignetten entwickelt (Gartmeier 2014; Hoppe-Seyler et al. 2014; Piwowar et al. 2017). Die Videofälle haben eine Länge von ca. zwei Minuten. In den insgesamt sechs Videofällen bearbeitet eine Gruppe von vier Schülerinnen und Schülern Aufgaben zu Stoffkreisläufen (je 3 Videofälle mit Aufgaben zu Pflanzen in Ökosystemen bzw. zur Stoffzersetzung). Dabei 


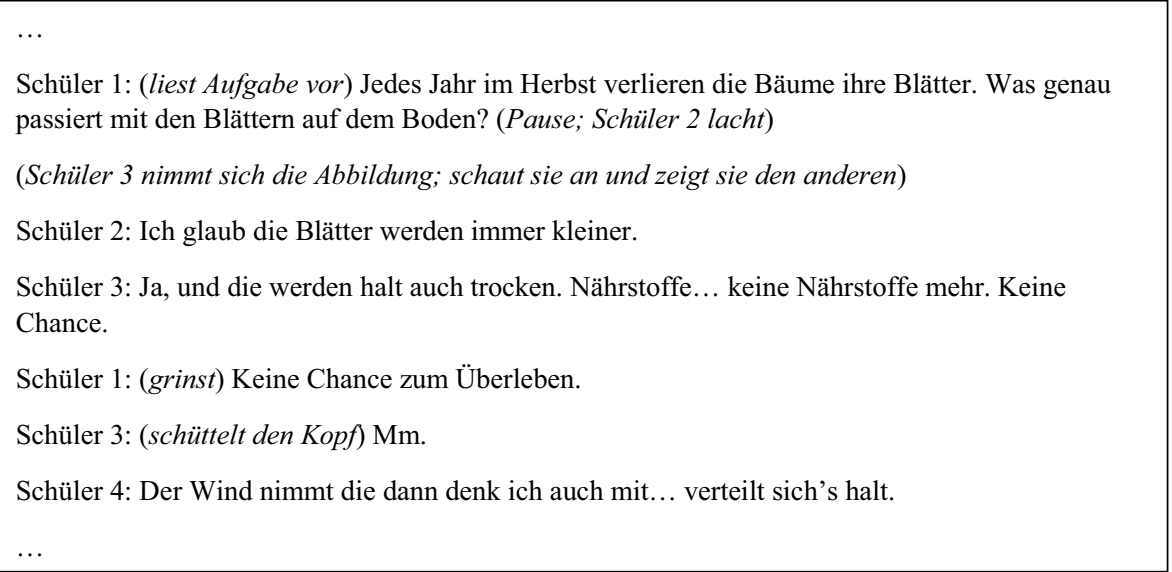

Abb. 1 Auschnitt aus einem Textfall. Gespräch zwischen Schülern über die Ursachen und Prozesse der Stoffzersetzung

werden jeweils Schülervorstellungen zur Bedeutung von Pflanzen in Ökosystemen (BP) bzw. Schülervorstellungen zur Stoffzersetzung (SZ) geäußert. Die Auswahl der Schülervorstellungen, welche in den Gruppenarbeiten geäußert werden, erfolgte literaturbasiert um möglichst repräsentative Fälle abzubilden (Dannemann et al. 2019; Piwowar et al. 2017; Schneider et al. 2016). Um vergleichbare Textfälle zu erstellen, wurden die Videofälle transkribiert und um nonverbale Informationen (Mimik/Gestik der Schüler etc.) angereichert. So entstanden sechs Fälle in Form von Transkripten mit je ca. 2000 Zeichen (s. Abb. 1). Sowohl Video- als auch Textfälle enthalten Zusatzinformationen zu Schulart, Klassenstufe, Thema der Stunde und verwendeten Unterrichtsmaterialien.

\subsection{Instrument zur Erfassung der Diagnosefertigkeiten}

Um die Wirkung der Intervention auf die Diagnosefertigkeiten zu erfassen, wurde sowohl vor als auch nach der Intervention ein Videotest durchgeführt (vgl. z. B. Blomberg et al. 2011). Zu beiden Messzeitpunkten wurde dasselbe Messinstrument verwendet. Der Videotest zur Messung der Diagnosefertigkeiten bestand aus zwei kurzen Videofällen (ca. 2 min) mit Schülerinnen und Schülern der neunten Klassenstufe einer Realschule. Eine Gruppe von vier Schülerinnen und Schülern löst Aufgaben zum Themenbereich der Stoffkreisläufe. Die Videofälle wurden in Anlehnung an authentische Unterrichtsszenen geskriptet. Die Unterrichtsausschnitte wurden so ausgewählt, dass die Äußerungen zu fachlichen Konzepten durch die Schülerinnen und Schüler im Fokus der Unterrichtsszenen stehen. Die Auswahl der Schülervorstellungen erfolgte mit Bezug zu vorliegenden Forschungsergebnissen, die in Zusammenstellungen typischer Schülervorstellungen aufgeführt werden (Hammann und Asshoff 2015; Kattmann 2015). Die in den Videofällen geäußerten Schülervorstellungen wurden jeweils den entsprechenden Schülervorstellungen zur Bedeutung von Pflanzen in Ökosystemen (erster Teil des Videotests, z. B. anthropo- 
zentrische Zuweisung von Bedeutung) bzw. Schülervorstellungen zur Stoffzersetzung (zweiter Teil des Videotests, z. B. Zersetzung durch Einwirkung abiotischer Faktoren) zugeordnet. Ein Beispiel zur Schülervorstellung Zersetzung durch Einwirkung abiotischer Faktoren (vgl. z. B. Hammann und Asshoff 2015) ist die Aussage eines Schülers, dass die Veränderungen bei den Blättern durch Wind verursacht würden (vgl. Schüler 4 im Transkript, s. Abb. 1).

$\mathrm{Zu}$ jedem Videofall erhielten die Studierenden Informationen zur Unterrichtssequenz (z.B. Namen der Schülerinnen und Schüler, Klassenstufe, verwendete Arbeitsmaterialien), um Beobachtungen einzelnen Lernenden zuordnen zu können und sich ein Bild von der diagnostischen Situation zu machen. Die im Test verwendeten Videofälle unterschieden sich von den Videofällen im Rahmen der Intervention sowohl in Bezug auf die Schülergruppe, als auch die Aufgaben, welche von den Schülerinnen und Schülern bearbeitet wurden.

Die Studierenden sollten die offene Frage „Was erscheint Ihnen aus der Perspektive eines Biologielehrers/einer Biologielehrerin relevant für den weiteren LehrLernprozess?" beantworten und ihre Beobachtungen bezugnehmend auf einzelne Schüleräußerungen mit kurzen Erläuterungen und mit entsprechenden Zeitankern notieren (Minuten- und Sekundenangabe). Für die Erfassung der Interpretationsleistung von Unterrichtssituationen und speziell auch von Schülervorstellungen in Schüleräußerungen hat sich ein offenes Antwortformat bewährt (Kersting 2008; Rath 2017). Durch dieses können verschiedene Niveaus von Diagnosefertigkeiten erfasst werden. Beispielsweise kann festgestellt werden, ob eine Schülervorstellung in einer Schüleräußerung wahrgenommen wird und inwiefern die Denkweise thematisch verortet und benannt werden kann. Durch die Formulierung der offenen Frage sollte abgebildet werden, dass sich unterrichtsbegleitendes Diagnostizieren häufig spontan und unmittelbar vollzieht (Shavelson et al. 2008). Durch die Verwendung von Videos sollte die Flüchtigkeit der diagnostischen Situation abgebildet werden (Leahy und Sweller 2011). Die Studierenden durften die Videofälle nur einmal anschauen und lediglich zum Notieren von Beobachtungen pausieren. Die Bewältigung der auf diese Weise präsentierten Anforderungen verlangt, dass schnell und situationsbezogen auf Professionswissen zurückgegriffen werden muss (vgl. Blömeke et al. 2014). Um die Reliabilität der Messung von Diagnosefertigkeiten mithilfe des Vignettentests zu bestimmen, wurde Cronbachs Alpha berechnet. Die Reliabilität war gut (Cronbachs Alpha =0,86).

\subsection{Versuchsablauf}

Einführend wurde allen Studierenden im Vorlesungsstil diagnoserelevantes Fachwissen (Ökosystem Wald, Biozönosen, Zersetzung etc.) und allgemeines fachdidaktisches Wissen zu Schülervorstellungen (z. B. Bedeutung von und Umgang mit Schülervorstellungen) in zwei Sitzungen à 90 min. vermittelt (s. Tab. 2). Spezifisches fachdidaktisches Wissen über typische Schülervorstellungen zum Themenbereich der Stoffkreisläufe wurde im Rahmen eines 90-minütigen Diagnosetrainings vermittelt. Die Diagnosetrainings wurde nach den Vorgaben des evidenzbasierten Modells Problemorientierten Lehrens und Lernens (Rieß und Mischo 2017) gestaltet. Zunächst wurde den Studierenden Vorgehensweisen und Lösungsbeispiele 
Tab. 2 Ablauf des Versuchs

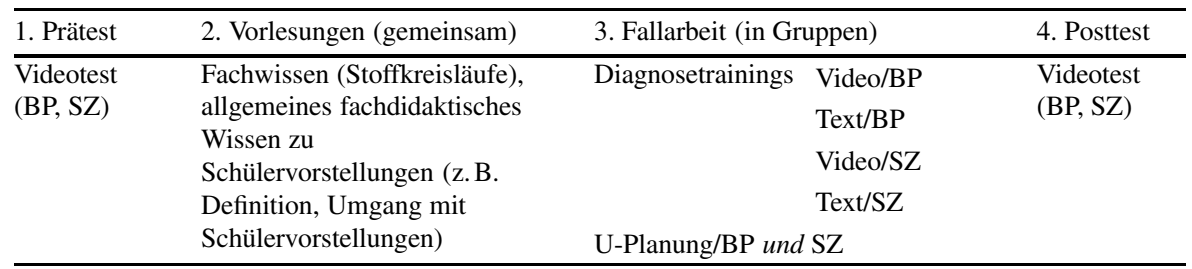

Im Rahmen der Vorlesungen wurde allen Teilnehmenden Fachwissen und allgemeines fachdidaktisches Wissen zu Schülervorstellungen vermittelt

Spezifisches fachdidaktisches Wissen zu den betreffenden Schülervorstellungen (BP bzw. SZ) wurde im Rahmen der Diagnosetrainings in Gruppen vermittelt

U-Planung (Kontrollgruppe) Unterrichtsplanung

„erfolgreicher“ Diagnostikerinnen und Diagnostiker gezeigt. Dabei wurden spezifische Schülervorstellungen mithilfe von Ankerbeispielen und treffenden Namen bzw. Beschreibungen nachvollziehbar gemacht (Schrenk et al. 2019). Anschließend wurden die Studierenden aufgefordert, insgesamt drei Video- oder Textfälle auf das Vorkommen von Schülervorstellungen hin zu analysieren, sich auszutauschen und ihre Antworten mit Lösungsvorschlägen zu vergleichen. Im Rahmen dieser Fallarbeit mit Hilfe von Videos bzw. Texten arbeiteten die Studierenden je nach Gruppenzuweisung entweder mit Schülervorstellungen zur Bedeutung von Pflanzen in Ökosystemen (BP) oder Schülervorstellungen zur Stoffzersetzung (SZ). Um den Einfluss der Dozierenden auf die Ergebnisse weitgehend auszuschließen sowie weitere Bedingungen (z. B. Lernzeit, Formulierung der Aufgaben) konstant zu halten, wurden diese fallbasierten Anteile der Intervention als vorstrukturierte Gruppenarbeit konzipiert und konnten von den Studierenden selbständig bearbeitet werden.

Die Sitzung einer Kontrollgruppe lief zeitlich parallel zu der Fallarbeitsphase der Interventionsgruppen ab. Der Kontrollgruppe wurde im Gegensatz zu den Interventionsgruppen spezifisches fachdidaktisches Wissen sowohl über Schülervorstellungen zur Bedeutung von Pflanzen in Ökosystemen (BP) also auch Schülervorstellungen zur Stoffzersetzung (SZ) vermittelt. Anstelle einer Anwendung dieses Wissens in Form von fallbasierten Diagnosetrainings erhielten die Studierenden die Aufgabe, einen Unterricht zu planen, in welchem diese Schülervorstellungen adressiert werden.

\subsection{Auswertung der Diagnoseleistung als abhängige Variable}

Die Diagnoseleistung (abhängige Variable) wird bestimmt durch die Ausprägung der Diagnosefertigkeiten des wissensgeleiteten Wahrnehmens und Interpretierens (s. Abschn. 2.3). Die Antworten der Studierenden wurden in Anlehnung an die qualitative Inhaltsanalyse (Mayring 2015) ausgewertet. Die Kategorienbildung erfolgte sowohl deduktiv als auch induktiv. Zunächst wurde kodiert, ob eine in Bezug auf Schülervorstellungen relevante Schüleräußerung überhaupt als bedeutsam für das weitere Lehren und Lernen erkannt wurde, d.h. ob die Studierenden eine Beobachtung zu der entsprechenden Stelle im Video mit einem Zeitanker notiert haben. Für diesen Fall wurde unterschieden, ob entweder in der betreffenden Schüleräußerung 
eine Schülervorstellung wahrgenommen wurde oder ob die Schüleräußerung in Bezug auf andere unterrichtliche Themenbereiche (z. B. Sozialverhalten) interpretiert wurde bzw. lediglich aus fachwissenschaftlicher Perspektive bewertet wurde. Wenn eine Schüleräußerung als Schülervorstellung wahrgenommen wurde, wurde kodiert, inwiefern die Schüleräußerung bezugnehmend auf die dahinterliegende Denkweise inhaltlich eingeschätzt wurde.

Insgesamt entstanden 18 Kategorien zur Beschreibung der Antworten durch die Studierenden. Diese wurden jeweils um Kodierregeln ergänzt. Für die Berechnung der Urteilerübereinstimmung anhand einer Übereinstimmungsmatrix wurden $10 \%$ der Daten doppelt kodiert. Für den Kodierleitfaden konnte eine gute Urteilerübereinstimmung gemessen werden $(\kappa=0,68)$. Die Kodierung der Daten erfolgte nach einem Beurteilertraining durch eine wissenschaftliche Hilfskraft.

Die im Rahmen der qualitativen Inhaltsanalyse gebildeten 18 Kategorien wurden in eine 6-stufige, durch drei Experten validierte $(\mathrm{ICC}=0,938)$, quasi-intervallskalierte Skala überführt, indem die Experten die Kategorien jeweils den Werten 1-6 zugeordnet haben $(1=$ sehr niedriges Niveau der Diagnosefertigkeiten, $6=$ sehr hohes Niveau der Diagnosefertigkeiten). Dem Wert 6 wurde z. B. die Kategorie Schülervorstellung wird korrekt interpretiert und sehr präzise kategorisiert zugeordnet. Jeder Antwort der Studierenden, die sich auf eine relevante Stelle im Video bezog, wurde entsprechend ein Wert von 1-6 zugewiesen. Die Werte wurden für jeden Teil des Videotests addiert und ergaben ausgehend von der Ausprägung der Diagnosefertigkeiten ein umfassendes Maß für die Diagnoseleistung in Form eines Summenscores. Die Diagnoseleistung entspricht hier dem Fertigkeitsniveau und bezieht sich auf beide hier erfassten, zusammenhängenden Teilfertigkeiten, also sowohl das bloße Wahrnehmen einer relevanten Schüleräußerung als auch eine in unterschiedlichem Maße angemessene Interpretation hinsichtlich der dahinterliegenden Schülervorstellung.

\section{Ergebnisse}

Tab. 3 zeigt die deskriptiven Werte für die wichtigsten Variablen in allen Experimentalbedingungen für beide Teile des Tests (Diagnostizieren von Schülervorstellungen zur Bedeutung von Pflanzen in Ökosystemen [BP] und Diagnostizieren von Schülervorstellungen zur Stoffzersetzung [SZ]). Allen Analysen wurde ein Signifikanzniveau von $5 \%$ zu Grunde gelegt. Die Signifikanztestung erfolgte zweiseitig. Als Maß für die Effektstärke nutzten wir das partielle $\eta^{2}$. Nach Cohen (1988) gelten Werte $<0,06$ als kleine Effekte, Werte zwischen 0,06 und 0,13 als mittlere Effekte und Werte von $>0,13$ als große Effekte. Ein mittlerer Effekt gilt für unsere Untersuchung als inhaltlich relevant. Aufgrund der verhältnismäßig kleinen Stichprobengröße wurden sämtliche Berechnungen auch mit nicht-parametrischen Verfahren durchgeführt.

\subsection{Voranalysen}

Die Gruppen unterschieden sich zum Prätestzeitpunkt nicht signifikant in ihren diagnostischen Fertigkeiten (Tab. 3) sowie in den demographischen Daten Alter, Ge- 
Tab. 3 Minimal- und Maximalwerte, Mittelwerte und Standardabweichung der Variable Diagnoseleistung in beiden Teilen des Tests (Diagnose von Schülervorstellungen zur Bedeutung von Pflanzen in Ökosystemen [BP] bzw. Schülervorstellungen zur Stoffzersetzung [SZ])

\begin{tabular}{|c|c|c|c|c|c|}
\hline Variable & Versuchsgruppe & $N$ & Minimum & Maximum & $M(S D)$ \\
\hline \multirow{6}{*}{$\begin{array}{l}\text { Diagnosele } \\
\mathrm{BP} \\
\text { (Prätest) }\end{array}$} & Video_BP & 8 & 3 & 14 & $6,50(3,74)$ \\
\hline & Text_BP & 7 & 0 & 16 & $8,29(5,44)$ \\
\hline & Video_SZ & 8 & 4 & 14 & $6,62(3,29)$ \\
\hline & Text_SZ & 6 & 0 & 12 & $5,00(4,47)$ \\
\hline & Kontrollgruppe/BP und SZ & 7 & 0 & 17 & $8,29(6,99)$ \\
\hline & Gesamt & 36 & 0 & 17 & $6,97(4,77)$ \\
\hline \multirow{6}{*}{$\begin{array}{l}\text { Diagnoseleistung } \\
\text { BP } \\
\text { (Posttest) }\end{array}$} & Video_BP & 8 & 19 & 37 & $26,50(5,98)$ \\
\hline & Text_BP & 7 & 15 & 40 & $24,71(10,29)$ \\
\hline & Video_SZ & 8 & 6 & 18 & $12,63(4,53)$ \\
\hline & Text_SZ & 6 & 5 & 20 & $12,17(5,49)$ \\
\hline & Kontrollgruppe/BP und SZ & 7 & 5 & 41 & $21,71(11,28)$ \\
\hline & Gesamt & 36 & 5 & 41 & $19,75(9,65)$ \\
\hline \multirow{6}{*}{$\begin{array}{l}\text { Diagnoseleistung } \\
\text { SZ } \\
\text { (Prätest) }\end{array}$} & Video_BP & 8 & 0 & 19 & $8,38(6,09)$ \\
\hline & Text_BP & 7 & 0 & 24 & $11,29(9,43)$ \\
\hline & Video/SZ & 8 & 4 & 31 & $11,75(9,91)$ \\
\hline & Text_SZ & 6 & 2 & 21 & $9,00(6,39)$ \\
\hline & Kontrollgruppe/BP und SZ & 7 & 0 & 33 & $14,57(11,36)$ \\
\hline & Gesamt & 36 & 0 & 33 & $11,00(8,67)$ \\
\hline \multirow{6}{*}{$\begin{array}{l}\text { Diagnoseleistung } \\
\text { SZ } \\
\text { (Posttest) }\end{array}$} & Video_BP & 8 & 0 & 27 & $14,87(9,20)$ \\
\hline & Text_BP & 7 & 8 & 24 & $16,00(5,23)$ \\
\hline & Video_SZ & 8 & 11 & 85 & $43,25(23,91)$ \\
\hline & Text_SZ & 6 & 36 & 89 & $57,00(17,86)$ \\
\hline & Kontrollgruppe/BP und SZ & 7 & 12 & 28 & $19,86(6,94)$ \\
\hline & Gesamt & 36 & 0 & 89 & $29,39(21,60)$ \\
\hline
\end{tabular}

Max. möglich ist ein Summenscore von 90 Punkten (BP) bzw. 108 Punkten (SZ)

schlechterverteilung, Studiengang (Primar- bzw. Sekundarstufe), Fachsemester und Benotung im Fach Biologie (alle $p s>0,20$ ).

\subsection{Forschungsfrage 1: Führt ein Training an Fallmedien in Kombination mit der Vermittlung spezifischen fachdidaktischen Wissens zu einer Verbesserung diagnostischer Fertigkeiten im Vergleich zu einer Kontrollgruppe, in der nur spezifisches fachdidaktisches Wissen vermittelt wird?}

Um diese Fragestellung zu untersuchen, wurden die Zuwächse der Diagnoseleistung von Prä- zu Posttest von Lehramtsstudierenden der Kontrollgruppe verglichen mit den Zuwächsen in der Diagnoseleistung bei Lehramtsstudierenden, die jeweils an video- bzw. textbasierten Diagnosetrainings zu einem bestimmten Inhalt (Bedeutung von Pflanzen in Ökosystemen [BP] oder Stoffzersetzung [SZ]) teilgenommen haben. Die deskriptiven Werte für die einzelnen Gruppen sind in Tab. 3 dargestellt. Abb. 2 

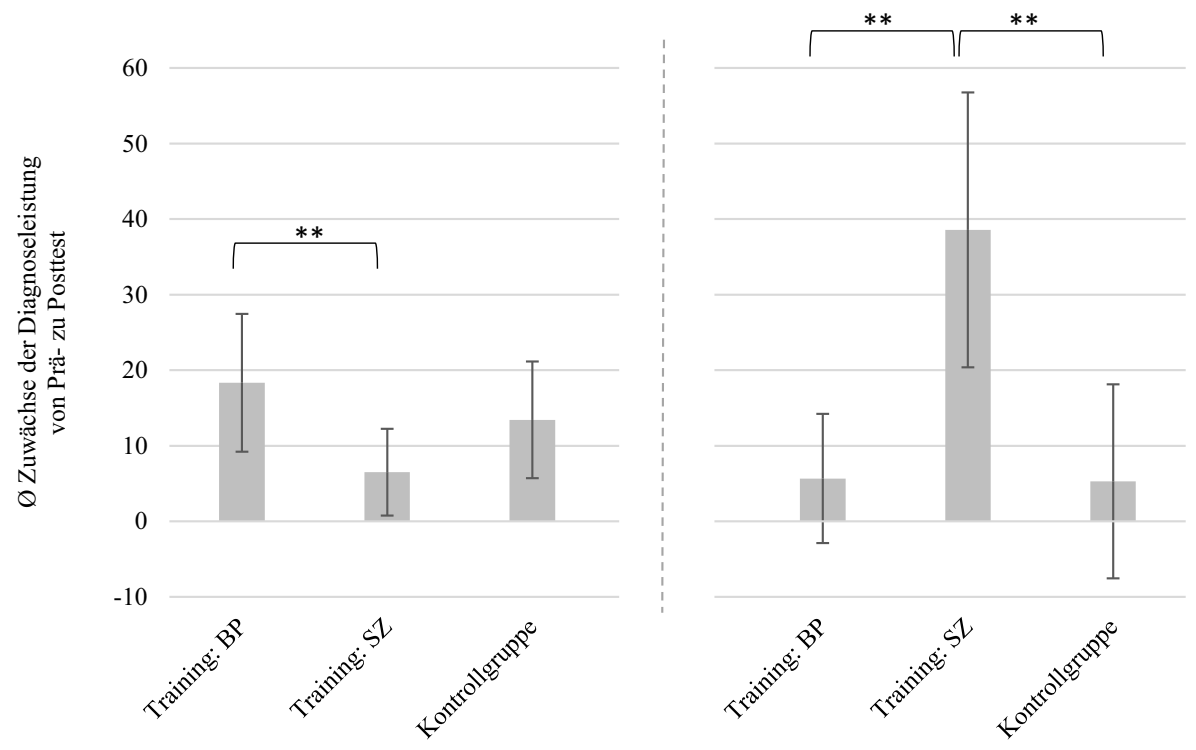

Abb. 2 Zuwächse der Diagnoseleistung von Prä- zu Posttest in beiden inhaltlichen Teile des Tests (BP und SZ) mit Standardabweichungen. BP bzw. SZ stehen für die jeweiligen Schülervorstellungen, welche Gegenstand der Tests bzw. der Diagnosetrainings waren (BP Bedeutung von Pflanzen, SZ Stoffzersetzung). Die fallmedienbasierten Trainingsgruppen umfassen die Versuchsgruppen Video_BP und Text_BP $(n=15)$ bzw. Video_SZ und Text_SZ $(n=14)$. Als Fallmedien wurden in den Trainings Videos oder Texte eingesetzt. In der Kontrollgruppe $(n=7)$ wurden Schülervorstellungen sowohl zu BP als auch SZ vermittelt, allerdings ohne Diagnosetrainings. Theoretisch war maximal möglich ein Summenscore im Wert von 108 Punkten. $(* p<0,05 ; * *<0,01)$

gibt einen Überblick über die Zuwächse der Diagnoseleistung von Prä- zu Posttest für beide Teile des Videotests (BP und SZ).

Eine ANOVA mit Messwiederholung zeigte für den ersten Teil des Tests (BP), dass es eine statistisch signifikante Interaktion zwischen der Zeit und den Untersuchungsgruppen gab, $F(2 ; 33)=8,62 ; p=0,001$; partielles $\eta^{2}=0,343$. Bei einem Vergleich mit der Kontrollgruppe, welcher fachdidaktisches Wissen ohne ein Diagnosetraining an Fallmedien vermittelt wurde, konnte gemäß einem Bonferronikorrigierten post-hoc-Test kein signifikanter Unterschied im Zuwachs der Diagnoseleistung festgestellt werden. Ein Training an Fallmedien hat sich für das Diagnostizieren von Schülervorstellungen zur Bedeutung von Pflanzen in Ökosystemen nicht als besonders wirksam erwiesen. Entscheidend schien dagegen der Inhaltsbereich zu sein, welcher Gegenstand des Diagnosetrainings war: Es zeigte sich, dass der Zuwachs der Diagnoseleistung bei der Gruppe, welche mit Schülervorstellungen zur Bedeutung von Pflanzen in Ökosystemen (BP) trainiert hat, deutlich höher ausfiel, als bei derjenigen Gruppe, die an einem Diagnosetraining zu einem anderen Thema (SZ) teilgenommen hat $(p=0,001)$.

Für den zweiten Teil des Tests (SZ), zeigte eine ANOVA mit Messwiederholung, dass es auch hier eine signifikante Interaktion zwischen der Zeit und den Untersu- 
chungsgruppen gab, $F(2 ; 33)=24,38 ; p<0,001$, partielles $\eta^{2}=596$. Gemäß einem Bonferroni-korrigierten post-hoc-Test unterschieden sich die Zuwächse in der Diagnoseleistung in der Gruppe, welche an Fallmedien zum Thema SZ trainiert hat, signifikant von der Kontrollgruppe $(p<0,001)$. Beim Diagnostizieren von Schülervorstellungen zur Stoffzersetzung (SZ) erhöhte sich also die Diagnoseleistung in den Gruppen, die an einem Diagnosetraining an Fallmedien teilgenommen haben deutlich im Vergleich zur Kontrollgruppe - allerdings nur dann, wenn auch mit dem entsprechenden Inhaltsbereich (SZ) trainiert wurde. Der Zuwachs der Diagnoseleistung in der Gruppe, welche an Fallmedien zum Thema SZ trainiert hat, fiel signifikant höher aus als bei der Gruppe, die an einem Diagnosetraining an Fallmedien zu einem anderen Thema (BP) teilgenommen hat $(p<0,001)$.

Zusammenfassend kann mit Bezug zu Forschungsfrage 1 festgehalten werden, dass ein Training an Fallmedien zu einer Verbesserung der diagnostischen Fertigkeiten führt verglichen mit einer Kontrollgruppe, der spezifisches fachdidaktisches Wissen vermittelt wird - allerdings nur bei einem bestimmten Themenbereich (SZ). Darüber hinaus deutet sich an, dass neben den Trainings an Fallmedien die Inhalte, mit denen trainiert wurde, einen entscheidenden Einfluss auf den Erwerb von Diagnosefertigkeiten zu haben scheinen. Dieser Befund wird unter Forschungsfrage 3 näher diskutiert.

\subsection{Forschungsfrage 2: Lassen sich diagnostische Fertigkeiten mit Video- oder Textvignetten wirksamer fördern?}

Um diese Fragestellung zu untersuchen, wurden die Zuwächse der Diagnoseleistung von Prä- zu Posttest von denjenigen Lehramtsstudierenden verglichen, die an Diagnosetrainings mithilfe von Videos bzw. Texten jeweils zu den gleichen biologischen Inhalten (BP bzw. SZ) teilgenommen haben. Bei einem Vergleich der Gruppen Video_BP und Text_BP konnten mithilfe von $t$-Tests weder für das Diagnostizieren von Schülervorstellungen zur Bedeutung von Pflanzen in Ökosystemen (BP) noch für das Diagnostizieren von Schülervorstellungen zur Stoffzersetzung (SZ) signifikante Unterschiede im Zuwachs der Diagnoseleistung ausgehend von der Verwendung unterschiedlicher Fallmedien festgestellt werden, $t(13)=0,75 ; p=0,470 \mathrm{bzw}$. $t(13)=0,391 ; p=0,702$. Auch bei einem Vergleich der Gruppen Video_SZ und Text_SZ konnte für keinen der beiden Themenbereiche (BP und SZ) mithilfe von $t$-Tests signifikante Unterschiede im Zuwachs der Diagnoseleistung nachgewiesen werden, $t(12)=-0,36 ; p=0,72$ bzw. $t(12)=-1,82 ; p=0,09$.

Während es für den Zuwachs der Diagnoseleistung zumindest für den Themenbereich SZ einen entscheidenden Unterschied machte, ob an Fallmedien trainiert wurde oder nicht, scheint hier die Wahl des Medienformats (Video oder Text) unerheblich zu sein. 


\subsection{Forschungsfrage 3: Werden diagnostische Fertigkeiten mithilfe von vignettenbezogenen Fördermaßnahmen inhaltsspezifisch erworben oder erfolgt auch eine Sensibilisierung für einen anderen Inhaltsbereich?}

Um festzustellen, inwiefern Diagnosefertigkeiten im Rahmen der vignettenbezogenen Diagnosetrainings inhaltsbezogen erworben werden, wurde mithilfe von $t$-Tests für jede Experimentalgruppe der Zuwachs der Diagnoseleistung bezogen auf Inhalte, die Gegenstand eines Diagnosetrainings waren (z.B. BP), verglichen mit der Transferleistung, also der Diagnosefertigkeiten bezogen auf einen anderen Inhaltsbereich (z. B. SZ). Die deskriptiven Werte für die Diagnoseleistung zum Prä- und zum Posttestzeitpunkt sind in Tab. 3 dargestellt.

Die Gruppe Video_BP hat an einem Diagnosetraining bezogen auf Schülervorstellungen zur Bedeutung von Pflanzen in Ökosystemen (BP) teilgenommen. Der Zuwachs der Diagnoseleistung war bezogen auf entsprechende Schülervorstellungen (BP) signifikant höher als bezogen auf Schülervorstellungen aus dem Bereich Stoffzersetzung, $t(7)=3,82 ; p=0,006$. Auch bei den anderen Gruppen, welche an Diagnosetrainings zu spezifischen Inhalten teilgenommen haben, konnte ein signifikant höherer Zuwachs der Diagnoseleistung festgestellt werden bezogen auf diejenigen Inhalte, welche Gegenstand der Fördermaßnahmen waren verglichen mit anderen Inhaltsbereichen. Die Signifikanzwerte lagen bei $p=0,039$ (Text_BP), $p=0,001$ (Video_SZ) und $p=0,002$ (Text_SZ). Im Vergleich zu den inhaltsspezifischen Fördermaßnahmen scheint eine Sensibilisierung und Förderung in einem anderen Inhaltsbereich für den Erwerb der hier fokussierten Diagnosefertigkeiten wenig wirksam zu sein.

\section{Diskussion}

In der vorliegenden Studie wurde untersucht, inwiefern sich eine Variation der Fallmedien zur Förderung von Diagnosefertigkeiten (Video- bzw. Textvignetten) und eine Variation der Diagnosegegenstände (unterschiedliche Schülervorstellungen) auf den Erwerb von Diagnosefertigkeiten von Lehramtsstudierenden in flüchtigen Unterrichtssituationen auswirken. Es wurden drei Forschungsfragen formuliert und experimentell untersucht.

\subsection{Führt ein Training an Fallmedien in Kombination mit der Vermittlung spezifischen fachdidaktischen Wissens zu einer Verbesserung diagnostischer Fertigkeiten im Vergleich zu einer Kontrollgruppe, in der nur spezifisches fachdidaktisches Wissen vermittelt wird?}

Beim Diagnostizieren von Schülervorstellungen zur Stoffzersetzung (SZ) zeigte sich, dass die Lehramtsstudierenden deutlich von einem Training an Fallmedien profitieren im Vergleich zu einer Vermittlung spezifischen fachdidaktischen Wissens ohne entsprechende Anwendungsmöglichkeit. Die Lehramtsstudierenden scheinen hier von einer Lerngelegenheit zu profitieren, die sich in wichtigen Facetten den Anfor- 
derungen gelingender unterrichtsbegleitender Diagnosen annähert (Grossman et al. 2018).

Beim Diagnostizieren von Schülervorstellungen zur Bedeutung von Pflanzen in Ökosystemen (BP) zeigte sich dieser Effekt nicht. Die Diagnosefertigkeiten der Lehramtsstudierenden verbesserten sich zwar, allerdings nicht signifikant im Vergleich zur Kontrollgruppe. Eine mögliche Ursache dafür, dass die Gruppen, welche mit Schülervorstellungen zur Bedeutung von Pflanzen in Ökosystemen gearbeitet haben, scheinbar weniger von den Trainings an Fallmedien profitiert haben, liegt in den unterschiedlich komplexen gedanklichen Konstruktionen der betreffenden Schülervorstellungen (BP und SZ) und damit unterschiedlichen Anforderungen beim Wahrnehmen und Interpretieren.

Um diese Annahme zu erklären, kann eine Analogie zu hoch- und niedrig-inferenten Urteilen bei der Unterrichtsdiagnostik gezogen werden (Schrader 2014). Während die in der Studie verwendeten Schülervorstellungen zur Stoffzersetzung (SZ) zu den weniger komplexen Konzepten gezählt werden können, handelt es sich bei den ausgewählten Schülervorstellungen zur Bedeutung von Pflanzen in Ökosystemen eher um komplexere Denkfiguren (Gropengießer 2006). Das Diagnostizieren von komplexen Schülervorstellungen (hier: Denkfiguren [BP]) ist ähnlich wie das hoch-inferente Urteilen insofern herausfordernd, als dass unterschiedliche Indikatoren in ihrem Zusammenhang und teilweise über längere Zeitabschnitte hinweg wahrgenommen und aufeinander bezogen werden müssen. Erst dadurch kann auf das Vorliegen einer bestimmten Schülervorstellung geschlossen werden. Dass dagegen weniger komplexe Schülervorstellungen (hier: Konzepte [SZ]) in einem einzigen Satz oder einer Behauptung ausgedrückt werden können (Gropengießer und Marohn 2018), könnte die Häufigkeit der Wahrnehmung und Interpretation von Schülervorstellungen und damit den Erwerb von Diagnosefertigkeiten bei Trainings an Fallmedien begünstigen.

\subsection{Lassen sich diagnostische Fertigkeiten mit Video- oder Textvignetten wirksamer fördern?}

Ein wesentliches Ziel der Studie war zu untersuchen, ob ein bestimmtes Fallmedium (Video oder Text) als Förderinstrument für ,on-the-fly“-Diagnosen von Schülervorstellungen eher geeignet ist. Ergebnisse bisheriger Studien deuten darauf hin, dass Video- und Textfälle bei der Förderung bestimmter Lehrerkompetenzen weitgehend gleichermaßen geeignet sind (Kramer et al. 2017; Schneider et al. 2016). Die Studie repliziert Ergebnisse vorausgegangener Studien insofern, als dass ein Training mit Textfällen ein ähnliches Potential zu haben scheint wie ein Training mit Videofällen. Weder für die Diagnose von Schülervorstellungen zur Bedeutung von Pflanzen (BP), noch für die Diagnose von Schülervorstellungen zur Stoffzersetzung (SZ) konnte bei der Untersuchung der Effekte eine unterschiedliche Wirkung der Lernbedingung Video vs. Text festgestellt werden. 


\subsection{Werden diagnostische Fertigkeiten mithilfe von vignettenbezogenen Fördermaßnahmen inhaltsspezifisch erworben oder erfolgt auch eine Sensibilisierung für einen anderen Inhaltsbereich?}

Das spezifische fachdidaktische Wissen (hier: Wissen über spezifische Schülervorstellungen), welches im Rahmen der Diagnosetrainings vermittelt wurde, scheint eine wesentliche Rolle für den Erwerb von Diagnosefertigkeiten zu spielen: Diagnostiziert werden diejenigen Schülervorstellungen, mit denen auch spezifisch trainiert wurde. Der Befund zu eingeschränkten Transferleistungen über verschiedene Arten von Schülervorstellungen hinweg unterstreicht die Bedeutung von spezifischem fachdidaktischen Wissen für den Erwerb von Diagnosefertigkeiten (siehe auch Todorova et al. 2017). Eine Sensibilisierung und Förderung in einem anderen Inhaltsbereich scheint in Bezug auf die hier untersuchten Diagnosefertigkeiten wenig zu bewirken. Diagnosefertigkeiten scheinen hier inhaltsspezifisch erworben zu werden.

\subsection{Implikationen für die Lehrerbildung}

Hinsichtlich des Erwerbs von Diagnosefertigkeiten scheint es im Allgemeinen nicht entscheidend zu sein, ob als Förderinstrumente Video- oder Textvignetten verwendet werden. Dazu ist anzumerken, dass für die verwendeten Schülervorstellungen und die betreffende diagnostische Situation fast ausschließlich verbale Äußerungen der Schülerinnen und Schüler von Bedeutung sind. Verbale Äußerungen können in Textvignetten (hier: Transkripten) gut abgebildet werden. Für andere Diagnoseinhalte bzw. diagnostische Situationen sind möglicherweise weitere Informationen wie der Einsatz von Experimenten oder Unterrichtsmedien bzw. Gestik und Mimik (z.B. bei Schüler-Lehrer-Interaktionen) relevant. In solchen Fällen liegt ggfs. ein Mehrwert in der Verwendung von Fallvideos, die die Unterrichtssituationen in ihrer Komplexität und Reichhaltigkeit umfassender als Textfälle abbilden (Koc et al. 2009).

Bei Schülervorstellungen, welche konkret auf sachliche Inhalten bezogen sind (hier: SZ), scheinen Lehramtsstudierende in Bezug auf die Förderung ihrer Diagnosefertigkeiten bereits von einer kurzen Trainingsintervention mithilfe von Fallmedien stark zu profitieren. Schneider et al. (2016) konnten feststellen, dass sich die Anzahl fallbasierter Sitzungen (mit Video oder Text) positiv auf die thematisch fokussierte Selektion von Situationen auswirkte. Für den Fall möglicherweise komplexerer Schülervorstellungen (hier: BP) ist entsprechend zu prüfen, ob ggfs. weitere Trainingseinheiten die Diagnosefertigkeiten verbessern.

Die Vermittlung von spezifischem fachdidaktischen Wissen, also Wissen zu spezifischen Schülervorstellungen, scheint grundlegend für gelingende Diagnosen zu sein. Da sich ein Transfer von Diagnosefertigkeiten zwischen unterschiedlichen Inhaltsdomänen schwierig gestaltet, profitieren Lehramtsstudierende möglicherweise, wenn möglichst viele und unterschiedliche Schülervorstellungen repräsentiert sind. Todorova et al. (2017) zogen für die Förderung professioneller Unterrichtswahrnehmung bezogen auf Lernerunterstützung im naturwissenschaftlichen Unterricht eine ähnliche Schlussfolgerung. Auch wenn die Möglichkeiten Transfers herzustellen 
begrenzt sind, dürften fallbasierte Diagnosetrainings für Lehramtsstudierende auch insofern in Bezug auf andere inhaltliche biologische Themenbereiche förderlich sein, als dass möglicherweise allgemeine Unterrichtsprinzipien - etwa die Notwendigkeit eines Perspektivenwechsels hin zur Schülerperspektive - vermittelt werden können (Dannemann et al. 2014).

\subsection{Grenzen der Studie und weitere Forschung}

In Bezug auf die Studie ist einschränkend zu bemerken, dass sich aufgrund der kleinen Stichprobe und der fehlenden (statistischen) Power bei der Auswertung der Ergebnisse lediglich Indizien hinsichtlich des Einflusses fallbasierter Diagnosetrainings sowie unterschiedlicher Schülervorstellungen auf den Erwerb von Diagnosefertigkeiten von Lehramtsstudierenden gewinnen lassen, es jedoch einer Replikation in einer größeren Studie bedarf.

Wir weisen zudem darauf hin, dass für den von uns eingesetzten Vignettentest neben den berichteten Reliabilitätskoeffizienten keine weiteren testtheoretischen Gütekriterien vorlagen. Aufgrund des spezifischen fachdidaktischen Kontexts, in dem Diagnosefertigkeiten hier gemessen wurden, konnten wir leider nicht auf Instrumente mit belegter Reliabilität und Validität zurückgreifen. Grundsätzlich hat sich aber die Erfassung von professionellen Fertigkeiten von Lehrkräften mithilfe von Videovignetten vielfach bewährt (z. B. Hoth et al. 2018; Kaiser et al. 2015; Kersting 2008; Knievel et al. 2015; Star et al. 2011). Nichtsdestoweniger sollte in weiteren Studien vor allem die Validität unserer Art der vignettenbasierten Erfassung von fachdidaktischen diagnostischen Fertigkeiten untersucht werden.

Darüber hinaus lassen sich durch eine Erweiterung des Testinstruments möglicherweise weiterführende Erkenntnisse über den Erwerb von Diagnosefertigkeiten gewinnen. Aufgrund der Bedeutung, die bestimmte Wissensbestände für das Diagnostizieren haben, ist es hier wünschenswert, ergänzend auch deklarative Wissensanteile als Moderatoren gezielt zu erfassen (vgl. z.B. Blömeke et al. 2014). Darüber hinaus wäre es womöglich aufschlussreich, neben Wissenskomponenten auch z.B. Einstellungen oder Motivation zu erfassen, welche als weitere Anteile eines umfassenden Konstrukts diagnostischer Kompetenz angesehen werden (vgl. z. B. Blömeke et al. 2015; Loibl et al. 2020; dieses Heft).

In der verwendeten Testsituation wurde die Flüchtigkeit der diagnostischen Situation als ein Merkmal komplexer Unterrichtspraxis durch einen entsprechenden Einsatz der Videofälle umgesetzt. Im Unterricht sind diagnostische Situationen unter anderem ggfs. auch durch Zeitdruck geprägt. Dieser lässt sich durch eine flüchtige Präsentation von Schüleräußerungen nur bedingt darstellen. Um Zeitdruck beim Diagnostizieren abzubilden, ist es sinnvoll, diese Komponente in die Gestaltung eines Testinstruments aufzunehmen (indem Antworten unter Zeitdruck gegeben werden, vgl. z. B. Knievel et al. 2015).

Im Rahmen des DiaCoM-Rahmenmodells von Loibl et al. (2020) lassen sich die Befunde im Sinne der Informationsverarbeitungsprozesse folgendermaßen deuten: Lehrkräfte diagnostizieren Vorstellungen von Schülerinnen und Schülern zu ökologischen Konzepten. Das hierzu nötige (fachdidaktische) diagnostische Wissen (als Personencharakteristik) wurde nur beim weniger komplexen Diagnosegegenstand 
wirksam, was auf die Bedeutung der Verfügbarkeit dieses Wissens hinweist. Die relative Flüchtigkeit der Information in Videos im Vergleich zu Textvignetten (als Situationscharakteristik) beeinflusste die Prozesse der Informationsverarbeitung jedoch nicht negativ. Aussagen darüber, inwieweit hier Prozesse des Gedächtnisses und der kognitiven und motivationalen Einflüsse der situativen Verankerung eine Rolle spielen, können im Rahmen des hier vorliegenden Designs allerdings nicht getroffen werden.

Förderung Das Projekt wurde im Rahmen des Forschungs- und Nachwuchskollegs DiaKom (Diagnostische Kompetenzen von Lehrkräften: Einflüsse, Struktur und Förderung) der Pädagogischen Hochschulen Freiburg und Heidelberg vom Ministerium für Wissenschaft, Forschung und Kunst Baden-Württemberg gefördert.

Funding Open Access funding provided by Projekt DEAL.

Open Access Dieser Artikel wird unter der Creative Commons Namensnennung 4.0 International Lizenz veröffentlicht, welche die Nutzung, Vervielfältigung, Bearbeitung, Verbreitung und Wiedergabe in jeglichem Medium und Format erlaubt, sofern Sie den/die ursprünglichen Autor(en) und die Quelle ordnungsgemäß nennen, einen Link zur Creative Commons Lizenz beifügen und angeben, ob Änderungen vorgenommen wurden.

Die in diesem Artikel enthaltenen Bilder und sonstiges Drittmaterial unterliegen ebenfalls der genannten Creative Commons Lizenz, sofern sich aus der Abbildungslegende nichts anderes ergibt. Sofern das betreffende Material nicht unter der genannten Creative Commons Lizenz steht und die betreffende Handlung nicht nach gesetzlichen Vorschriften erlaubt ist, ist für die oben aufgeführten Weiterverwendungen des Materials die Einwilligung des jeweiligen Rechteinhabers einzuholen.

Weitere Details zur Lizenz entnehmen Sie bitte der Lizenzinformation auf http://creativecommons.org/ licenses/by/4.0/deed.de.

\section{Literatur}

von Aufschnaiter, C., Münster, C., \& Beretz, A.-K. (2018). Zielgerichtet und differenziert diagnostizieren. Der Mathematische und naturwissenschaftliche Unterricht, 71(6), 382-386.

Baisch, P. (2009). Schülervorstellungen zum Stoffkreislauf: Eine Interventionsstudie im Kontext einer Bildung für nachhaltige Entwicklung. Hamburg: Verlag Dr. Kovač.

Baumert, J., \& Kunter, M. (2006). Stichwort: Professionelle Kompetenz von Lehrkräften. Zeitschrift für Erziehungswissenschaft, 9(4), 469-520. https://doi.org/10.1007/s11618-006-0165-2.

Beck, E., Baer, M., Guldimann, T., Bischoff, S., \& Brühwiler, C. (2008). Adaptive Lehrkompetenz: Analyse und Struktur Veränderbarkeit und Wirkung handlungssteuernden Lehrerwissens. Pädagogische Psychologie und Entwicklungspsychologie, Bd. 63. Münster, München, Berlin: Waxmann.

Blomberg, G., Stürmer, K., \& Seidel, T. (2011). How pre-service teachers observe teaching on video: effects of viewers' teaching subjects and the subject of the video. Teaching and Teacher Education, 27(7), 1131-1140.

Blömeke, S., Gustafsson, J.-E., \& Shavelson, R. J. (2015). Beyond dichotomies. Zeitschrift für Psychologie, 223(1), 3-13.

Blömeke, S., König, J., Busse, A., Suhl, U., Benthien, J., Döhrmann, M., \& Kaiser, G. (2014). Von der Lehrerausbildung in den Beruf - Fachbezogenes Wissen als Voraussetzung für Wahrnehmung, Interpretation und Handeln im Unterricht. Zeitschrift für Erziehungswissenschaft, 17(3), 509-542.

Brühwiler, C. (2017). Diagnostische und didaktische Kompetenz als Kern adaptiver Lehrkompetenz. In A. Südkamp \& A.-K. Praetorius (Hrsg.), Pädagogische Psychologie und Entwicklungspsychologie. Diagnostische Kompetenz von Lehrkräften: Theoretische und methodische Weiterentwicklungen. Münster, New York: Waxmann.

Chernikova, O., Heitzmann, N., Fink, M.C., Timothy, V., Seidel, T., \& Fischer, F. (2019). Facilitating diagnostic competences in higher education-A meta-analysis in medical and teacher education. Educational Psychology Review, 68(1), 1-40. https://doi.org/10.1007/s10648-019-09492-2. 
Cohen, J. (1988). Statistical power analysis for behavioral sciences. Hillsdale, NJ: Lawrence Erlbaum.

Dannemann, S., Heeg, J., \& Schanze, S. (2019). Fallbasierte Förderung der Diagnose- und Planungsfähigkeiten von Lehramtsstudierenden. Lernen mit Videovignetten in der Biologie- und Chemiedidaktik. In E. Christophel, M. Hemmer, F. Korneck, T. Leuders \& P. Labudde (Hrsg.), Fachdidaktische Forschungen, Fachdidaktische Forschung zur Lehrerbildung (Bd. 11, S. 75-86). Münster, New York: Waxmann.

Dannemann, S., Meier, M., Hilfert-Rüppell, D., Kuhlemann, B., Eghtessad, A., Höner, K., Hößle, C., \& Looß, M. (2018). Erheben und Fördern der Diagnosekompetenz von Lehramtsstudierenden durch den Einsatz von Vignetten. In M. Hammann \& M. Lindner (Hrsg.), „Biologiedidaktik als Wissenschaft“: 21. Internationale Tagung der Fachsektion Didaktik der Biologie im VBIO, Martin-Luther-Universität Halle-Wittenberg, 2017. Lehr- und Lernforschung in der Biologiedidaktik, (Bd. 8, S. 245-266). Innsbruck, Wien, Bozen: Studien Verlag.

Dannemann, S., Niebert, K., Affeldt, S., \& Gropengießer, H. (2014). Fallsammlung zum Lehren und Lernen der Biologie - Entwicklung von Videovignetten. In I. Baumgardt (Hrsg.), Fachdidaktische Beiträge aus der universitären Praxis. Forschen, Lehren und Lernen in der Lehrerausbildung: Fachdidaktische Beiträge aus der universitären Praxis (S. 41-56). Baltmannsweiler: Schneider Hohengehren.

Decristan, J., Hondrich, A.L., Büttner, G., Hertel, S., Klieme, E., Kunter, M., Lühken, A., Adl-Amini, K., Djakovic, S., Mannel, S., Naumann, A., \& Hardy, I. (2015). Impact of additional guidance in science education on primary students' conceptual understanding. The Journal of Educational Research, 108(5), 358-370.

Duit, R., Treagust, D., \& Widoro, A. (2008). Teaching science for conceptual change: theory and practice. In S. Vosniadou (Hrsg.), International handbook of research on conceptual change (S. 629-645). London: Routledge.

Furtak, E. M., Kiemer, K., Circi, R. K., Swanson, R., de León, V., Morrison, D., \& Heredia, S. C. (2016a). Teachers' formative assessment abilities and their relationship to student learning: findings from a four-year intervention study. Instructional Science, 44(3), 267-291.

Furtak, E. M., Thompson, J., \& van Es, E. A. (2016b). Formative assessment and noticing: toward a synthesized framework for attending and responding during instruction. Annual Meeting of the American Educational Research Association, Washington, D.C., 9. Apr. 2016.

Gartmeier, M. (2014). Fiktionale Videofälle in der Lehrerinnen- und Lehrerbildung. Beiträge zur Lehrerinnen- und Lehrerbildung, 32(2), 235-246.

Gaudin, C., \& Chaliès, S. (2015). Video viewing in teacher education and professional development: a literature review. Educational Research Review, 16, 41-67.

Gebhard, U. (2013). Schülerinnen und Schüler. In H. Gropengießer, U. Harms \& U. Kattmann (Hrsg.), Fachdidaktik Biologie: Die Biologiedidaktik (9. Aufl. S. 198-211). Hallbergmoos: Aulis.

Glogger-Frey, I., \& Herppich, S. (2017). Formative Diagnostik als Teilaspekt diagnostischer Kompetenz. In A. Südkamp \& A.-K. Praetorius (Hrsg.), Pädagogische Psychologie und Entwicklungspsychologie. Diagnostische Kompetenz von Lehrkräften: Theoretische und methodische Weiterentwicklungen (S. 42-45). Münster, New York: Waxmann.

Gropengießer, H. (1996). Die Bilder im Kopf. Von den Vorstellungen der Lernenden ausgehen. Friedrich Jahresheft. (S. 11-13).

Gropengießer, H. (2006). Wie man Vorstellungen der Lerner verstehen kann: Lebenswelten, Denkwelten, Sprechwelten (2. Aufl.). Beiträge zur didaktischen Rekonstruktion, Bd. 4. Oldenburg: Didaktisches Zentrum Carl-von-Ossietzky-Univ.

Gropengießer, H., \& Marohn, A. (2018). Schülervorstellungen und Conceptual Change. In D. Krüger, I. Parchmann \& H. Schecker (Hrsg.), Theorien in der naturwissenschaftsdidaktischen Forschung (S. 49-66). Heidelberg, Berlin, New York: Springer.

Grossman, P., Compton, C., Igra, D., Ronfeldt, M., Shahan, E., \& Williamson, P. (2009). Teaching practice: a cross-professional perspective. Teachers College Record, 111(9), 2055-2100.

Grossman, P.L., Scheider Kavanagh, S., \& Pupik Dean, C.G. (2018). The turn towards practice-based teacher education: introduction to the work of the core practice consortium. In P. L. Grossman (Hrsg.), Teaching core practices in teacher education (S. 1-14). Cambridge: Harvard Education Press.

Hammann, M., \& Asshoff, R. (2015). Schülervorstellungen im Biologieunterricht: Ursachen für Lernschwierigkeiten (2. Aufl.). Seelze: Klett Kallmeyer.

Hammann, M., \& Jördens, J. (2014). Offene Aufgaben codieren. In D. Krüger, I. Parchmann \& H. Schecker (Hrsg.), Methoden in der naturwissenschaftsdidaktischen Forschung (S. 169-178). Heidelberg, Berlin, New York: Springer.

Häußler, P. (1998). Perspektiven für die Unterrichtspraxis: Naturwissenschaftsdidaktische Forschung. Kiel: IPN. 
Heitzmann, N., Seidel, T., Hetmanek, A., Wecker, C., Fischer, M., Ufer, S., Schmidmaier, R., Neuhaus, B., Siebeck, M., Stürmer, K., Obersteiner, A., Reiss, K., Girwidz, R., Fischer, F., \& Opitz, A. (2019). Facilitating diagnostic competences in simulations in higher education: a framework and a research agenda. Frontline Learning Research, 7(4), 1-24.

Helmke, A. (2017). Unterrichtsqualität und Lehrerprofessionalität (7. Aufl.). Seelze: Klett Kallmeyer.

Herppich, S., Praetorius, A.-K., Hetmanek, A., Glogger-Frey, I., Ufer, S., Leutner, D., Behrmann, L., Böhmer, I., Böhmer, M., Förster, N., Kaiser, J., Karing, C., Karst, K., Klug, J., Ohle, A., \& Südkamp, A. (2017a). Ein Arbeitsmodell für die empirische Erforschung der diagnostischen Kompetenz von Lehrkräften. In A. Südkamp \& A.-K. Praetorius (Hrsg.), Pädagogische Psychologie und Entwicklungspsychologie. Diagnostische Kompetenz von Lehrkräften: Theoretische und methodische Weiterentwicklungen (S. 75-93). Münster, New York: Waxmann.

Herppich, S., Praetorius, A.-K., Förster, N., Glogger-Frey, I., Karst, K., Leutner, D., Behrmann, L., Böhmer, M., Ufer, S., Klug, J., Hetmanek, A., Ohle, A., Böhmer, I., Karing, C., Kaiser, J., \& Südkamp, A. (2017b). Teachers' assessment competence: Integrating knowledge-, process-, and product-oriented approaches into a competence-oriented conceptual model. Teaching and Teacher Education. https:// doi.org/10.1016/j.tate.2017.12.001.

Hoppe-Seyler, T., Gartmeier, M., Möller, G., Bauer, J., Wiesbeck, A., \& Karsten, G. (2014). Entwicklung von Lehrfilmen zur Gesprächsführung zwischen Realitätsnähe und systematischer didaktischer Gestaltung. Zeitschrift für Hochschulentwicklung. https://doi.org/10.3217/zfhe-9-03/14.

Hoth, J., Kaiser, G., Döhrmann, M., König, J., \& Blömeke, S. (2018). A situated approach to assess teachers' professional competencies using classroom videos. In O. Buchbinder \& S. Kuntze (Hrsg.), ICME-13 monographs. Mathematics teachers engaging with representations of practice: a dynamically evolving field (S. 23-45). Cham: Springer.

Kaiser, G., Busse, A., Hoth, J., König, J., \& Blömeke, S. (2015). About the complexities of video-based assessments: Theoretical and methodological approaches to overcoming shortcomings of research on teachers' competence. International Journal of Science and Mathematics Education, 13(2), 369-387. https://doi.org/10.1007/s10763-015-9616-7.

Kattmann, U. (2015). Schüler besser verstehen: Alltagsvorstellungen im Biologieunterricht. Hallbergmoos: Aulis.

Kattmann, U. (2017). Biologie unterrichten mit Alltagsvorstellungen: Didaktische Rekonstruktion in Unterrichtseinheiten (1. Aufl.). Seelze: Klett Kallmeyer.

Kersting, N. (2008). Using video clips of mathematics classroom instruction as item prompts to measure teachers' knowledge of teaching mathematics. Educational and Psychological Measurement, 68(5), $845-861$.

Klieme, E., \& Warwas, J. (2011). Konzepte der Individuellen Förderung. Zeitschrift für Pädagogik, 57(6), 805-818.

Knievel, I., Lindmeier, A. M., \& Heinze, A. (2015). Beyond knowledge: measuring primary teachers' subject-specific competences in and for teaching mathematics with items based on video vignettes. International Journal of Science and Mathematics Education, 13(2), 309-329.

Koc, Y., Peker, D., \& Osmanoglu, A. (2009). Supporting teacher professional development through online video case study discussions: An assemblage of preservice and Inservice teachers and the case teacher. Teaching and Teacher Education, 25(8), 1158-1168.

Kramer, C., König, J., Kaiser, G., Ligtvoet, R., \& Blömeke, S. (2017). Der Einsatz von Unterrichtsvideos in der universitären Ausbildung: Zur Wirksamkeit video- und transkriptgestützter Seminare zur Klassenführung auf pädagogisches Wissen und situationsspezifische Fähigkeiten angehender Lehrkräfte. Zeitschrift für Erziehungswissenschaft, 20(S1), 137-164.

Krammer, K. (2014). Fallbasiertes Lernen mit Unterrichtsvideos in der Lehrerinnen- und Lehrerbildung. Beiträge zur Lehrerinnen- und Lehrerbildung, 32(2), 164-175.

Leach, J., Driver, R., Scott, P., \& Wood-Robinson, C. (2008). Children's ideas about ecology 2: Ideas found in children aged 5-16 about the cycling of matter. International Journal of Science Education, 18(1), 19-34.

Leahy, W., \& Sweller, J. (2011). Cognitive load theory, modality of presentation and the transient information effect. Applied Cognitive Psychology, 25(6), 943-951.

Loibl, K., Leuders, T., \& Dörfler, T. (2020). A framework for explaining teachers' diagnostic judgements by cognitive modeling (DiacoM). Teaching and Teacher Education, 91, 103059.

Mayring, P. (2015). Qualitative Inhaltsanalyse: Grundlagen und Techniken. Weinheim, Basel: Beltz.

Piwowar, V., Barth, V.L., Ophardt, D., \& Thiel, F. (2017). Evidence-based scripted videos on handling student misbehavior: The development and evaluation of video cases for teacher education. Professional Development in Education, 10(2), 1-16. 
Rath, V. (2017). Diagnostische Kompetenz von angehenden Physiklehrkräften. Berlin: Logos.

Reh, S., \& Schelle, C. (2010). Der Fall im Lehrerstudium. In C. Schelle, K. Rabenstein \& S. Reh (Hrsg.), Unterricht als Interaktion: Ein Fallbuch für die Lehrerbildung (S. 13-24). Bad Heilbrunn: Klinkhardt.

Riemeier, T. (2007). Moderater Konstruktivismus. In D. Krüger \& H. Vogt (Hrsg.), Theorien in der biologiedidaktischen Forschung (S. 69-79). Heidelberg, Berlin, New York: Springer.

Rieß, W., \& Mischo, C. (2017). „Bridging the gap“ - Zur Verringerung der Kluft zwischen allgemeinen Lehr-Lernmodellen und konkreter Unterrichtsgestaltung am Beispiel der Förderung dynamischer Problemlösekompetenz in der Biologie. Zeitschrift für Didaktik der Biologie (ZDB) - Biologie Lehren und Lernen, 21(1), 1-22.

Ruiz-Primo, M. A. (2011). Informal formative assessment: The role of instructional dialogues in assessing students' learning. Studies in Educational Evaluation, 37(1), 15-24.

Ruiz-Primo, M.A., \& Furtak, E. M. (2006). Informal formative assessment and scientific inquiry: Exploring teachers' practices and student learning. Educational Assessment, 11(3 \& 4), 205-235.

Ruiz-Primo, M. A., \& Furtak, E. M. (2007). Exploring teachers' informal formative assessment practices and students' understanding in the context of scientific inquiry. Journal of Research in Science Teaching, 44(1), 57-84.

Schecker, H., \& Duit, R. (2018). Schülervorstellungen und Physiklernen. In H. Schecker, T. Wilhelm, M. Hopf \& R. Duit (Hrsg.), Schülervorstellungen und Physikunterricht (S. 1-21). Heidelberg, Berlin, New York: Springer.

Schneider, J., Bohl, T., Kleinknecht, M., Rehm, M., Kuntze, S., \& Syring, M. (2016). Unterricht analysieren und reflektieren mit unterschiedlichen Fallmedien: Ist Video wirklich besser als Text? Unterrichtswissenschaft, 44(4), 474-490.

Schrader, F.-W. (2013). Diagnostische Kompetenz von Lehrpersonen. Beiträge zur Lehrerbildung, 31(2), 154-165.

Schrader, F.-W. (2014). Lehrer als Diagnostiker. In E. Terhart (Hrsg.), Handbuch der Forschung zum Lehrerberuf (2. Aufl. S. 883-904). Münster: Waxmann.

Schrader, F.-W., \& Praetorius, A.-K. (2018). Diagnostische Kompetenz von Eltern und Lehrern. In D. H. Rost, J. R. Sparfeldt \& S. Buch (Hrsg.), Handwörterbuch Pädagogische Psychologie (5. Aufl. S. 92-98). Weinheim, Basel: Beltz.

Schrenk, M., Gropengießer, H., Groß, J., Hammann, M., Weitzel, H., \& Zabel, J. (2019). Schülervorstellungen im Biologieunterricht. In J. Groß, M. Hammann, P. Schmiemann \& J. Zabel (Hrsg.), Biologiedidaktische Forschung: Erträge für die Praxis (S. 3-20). Heidelberg, Berlin, New York: Springer.

Schutz, K. M., Grossman, P., \& Shaughnessy, M. (2018). Approximations of practice in teacher education. In P. L. Grossman (Hrsg.), Teaching core practices in teacher education (S. 57-84). Cambridge: Harvard Education Press.

Shavelson, R. J., Yin, Y., Furtak, E. M., Ruiz-Primo, M. A., Ayala, C.C., Young, D. B., Tomita, M., Brandon, P., \& Pottenger, F. M. III (2008). On the role and impact of formative assessment on science inquiry teaching and learning. In Assessing science learning: perspectives from research \& practice (S. 21-36).

Sherin, M. G., Russ, R. S., \& Colestock, A. A. (2011). Accessing mathematics teachers' in-the-moment noticing. In M. G. Sherin (Hrsg.), Studies in mathematical thinking and learning. Mathematics teacher noticing: Seeing through teachers' eyes (S. 79-94). London: Routledge.

Spinath, B. (2005). Akkuratheit der Einschätzung von Schülermerkmalen durch Lehrer und das Konstrukt der diagnostischen Kompetenz. Zeitschrift für Pädagogische Psychologie, 19(1/2), 85-95.

Star, J. R., Lynch, K., \& Perova, N. (2011). Using video to improve Preservice mathematics teachers' abilities to attend to classroom features: a replication study. In M. G. Sherin (Hrsg.), Studies in mathematical thinking and learning. Mathematics teacher noticing: seeing through teachers' eyes (S. 117-133). London: Routledge.

Sunder, C., Todorova, M., \& Möller, K. (2016). Kann die professionelle Unterrichtswahrnehmung von Sachunterrichtsstudierenden trainiert werden? - Konzeption und Erprobung einer Intervention mit Videos aus dem naturwissenschaftlichen Grundschulunterricht. Zeitschrift für Didaktik der Naturwissenschaften, 22(1), 1-12.

Syring, M., Bohl, T., Kleinknecht, M., Kuntze, S., Rehm, M., \& Schneider, J. (2015). Videos oder Texte in der Lehrerbildung? Effekte unterschiedlicher Medien auf die kognitive Belastung und die motivational-emotionalen Prozesse beim Lernen mit Fällen. Zeitschrift für Erziehungswissenschaft, 18(4), $667-685$.

Taber, K. S. (2017). The nature of student conceptions. In K. S. Taber \& B. Akpan (Hrsg.), Science education: An international course companion. New directions in mathematics and science education, (Bd. 31, S. 119-131). Rotterdam, Boston, Taipei: Sense. 
Todorova, M., Sunder, C., Steffensky, M., \& Möller, K. (2017). Pre-service teachers' professional vision of instructional support in primary science classes: how content-specific is this skill and which learning opportunities in initial teacher education are relevant for its acquisition? Teaching and Teacher Education, 68, 275-288.

Van de Pol, J., Volman, M., Oort, F., \& Beishuizen, J. (2014). Teacher scaffolding in small-group work: an intervention study. Journal of the Learning Sciences, 23(4), 600-650.

Zabel, J., \& Gropengießer, H. (2011). Learning progress in evolution theory: Climbing a ladder or roaming a landscape? Journal of Biological Education, 45(3), 143-149. 